\title{
LAS ACTIVIDADES DE ANDINISMO EN LA FORMACIÓN DEL PROFESORADO EN EDUCACIÓN FÍSICA
}

\author{
THE ACTIVITIES OF MOUNTANEERING IN FORMATION OF PHYSICAL EDUCATION \\ PROFESSORSHIP
}

\author{
López, Eduardo \& Fernández, Marisa.
}

Centro Regional Universitario Bariloche. Universidad Nacional del Comahue, Bariloche, República Argentina.

LÓPEZ E. \& FERNÁNDEZ M. Las actividades de andinismo en la formación del profesorado en educación física Rev. Motr. Hum., 11(1): 6-10, 2010.

\begin{abstract}
RESUMEN
El trabajo describe algunos desafíos y problemáticas que se presentan en la formación del profesorado en Educación Física del Centro Regional Universitario Bariloche, dependiente de la Universidad Nacional del Comahue, Argentina; a partir de demandas vinculadas con el entorno natural. Esta carrera incluye espacios curriculares que promueven la construcción de la corporeidad y motricidad desde prácticas corporales culturalmente relevantes en el medio natural; espacio que resulta un potencial motivador, contribuye a la formación integral a partir de un abordaje transdisciplinario y promueve la autonomía de los sujetos en tanto exige toma de decisiones constantes en relación con sus características particulares. El medio natural brinda amplias posibilidades motrices que superan movimientos estereotipados, favorece el uso creativo del cuerpo y habilita nuevos ámbitos de intervención pedagógica.

Las actividades de Andinismo se han ido consolidando como contenidos curriculares, dando a esta formación docente universitaria características particulares en estrecha relación con la región.
\end{abstract}

Palabras Clave: Andinismo, medio natural, educación física, formación docente.

\section{DISEÑO CURRICULAR DEL PROFESORADO EN EDUCACIÓN FÍSICA}

En las últimas décadas, las actividades físicas en medios naturales y las prácticas de escalada en rocódromos se han desarrollado y extendido a nivel mundial $(1,2)$. Paulatinamente, se han reconocido los aportes que realizan en particular las actividades de escalada en relación con las potencialidades del trepar como cualidad básica del movimiento.

Estas prácticas, promovidas tanto en medios naturales o artificiales como en el ámbito de la educación no formal, se encuentran incorporadas como deportes escolares en el currículo de algunos países europeos con tradición en el montañismo. En nuestro país, en la provincia de Río Negro, la Versión Preliminar del Diseño Curricular para el Ciclo Básico de Escuela Secundaria, en proceso de implementación desde el año 2008, incluye como contenido de Educación Física en el eje temático "El adolescente y las situaciones motrices en el medio ambiente natural", a las prácticas de actividades con cuerdas, puentes, tirolesas, rapel y toperope.
La incorporación de estos contenidos expone nuevos desafíos y problemáticas en la formación docente de los profesores de Educación Física.

La carrera del Profesorado en Educación Física del Centro Regional Universitario Bariloche (CRUB), dependiente de la Universidad Nacional del Comahue, se inicia en el año 1991 y es la única en Latinoamérica que propone una orientación específica en actividades de montaña. Las asignaturas que conforman este trayecto de formación orientado son: Actividades en el Entorno Regional (anual, 120 horas, segundo año); Deportes Regionales Estivales I (anual, 150 horas, tercer año); Deportes Regionales Estivales II (anual, 150 horas, cuarto año). Estas materias y sus contenidos han sido construidos y reconstruidos a partir de las experiencias y propuestas pedagógicas implementadas a lo largo de los dieciocho años y de las necesarias revisiones y modificaciones de planes de estudios efectuadas. Dicho diseño curricular, en tanto proyecto político-pedagógico (3) que orienta la formación, se define y redefine en las prácticas cotidianas de los actores y requiere ser sometido a análisis constante en su dinámica y en su devenir. 
LÓPEZ E. \& FERNÁNDEZ M. Las actividades de andinismo en la formación del profesorado en educación física Rev. Motr. Hum.,

11(1): 6-10, 2010.

Tal como afirma Bracht, la Educación Física posee o reúne diversas prácticas con diversos significados y sentidos (4). El plan de estudios vigente la define como práctica pedagógica que se ocupa de la enseñanza de diferentes prácticas corporales reconocidas en la cultura, organizadas como contenidos, que contribuye al desarrollo integral de los sujetos cuyos ejes fundamentales son cuerpo y movimiento. El ejercicio pedagógico en el campo de la Educación Física, en tanto práctica social, ética y política, es caracterizado en el Diseño Curricular por su complejidad y por las múltiples dimensiones que lo atraviesan. Diversas tareas son requeridas en contextos variados que las condicionan, aunque no las determinan. En la práctica cotidiana se presentan situaciones simultáneas que exigen tomas de decisiones constantes, cuyos resultados no pueden anticiparse ni preverse certeramente. La racionalidad técnica no resulta suficiente, por lo que se requieren prácticas reflexivas que den lugar a la revisión de lo realizado, que permitan volver a mirar lo actuado y pensado; no desde la simple contemplación, sino que es necesario remitirse hacia el análisis y la consideración de lo que ocurre en la experiencia diaria para planificar y concretar propuestas alternativas. La reflexión favorece el fortalecimiento de la capacidad de decisión y es el anclaje para la transformación. Implica un compromiso a explorar experiencias para lograr mejores comprensiones y apreciaciones. Los procesos reflexivos demandan tiempo y dispositivos específicos para poner en palabras las prácticas desarrolladas. En este sentido, no es episódica; sino que exige una mirada profunda de las propuestas implementadas. El plan de estudios propone una Educación Física que pueda incluir e incorporar propuestas formativas que den respuesta a las variadas y cambiantes demandas sociales y que contribuyan a la construcción de la corporeidad y motricidad, fomentando espacios de apropiación de la cultura corporal. Por otro lado, la región y el territorio nacional promueven desde las características de su entorno, la necesidad de formación de profesores capaces de desempeñarse en actividades adecuadas a los desafíos del medio natural.

\section{¿MONTAÑISMO, ANDINISMO, ALPINISMO?}

No existe consenso internacional para definir a las actividades de Andinismo, Alpinismo o Montañismo. Algunos autores afirman que el Montañismo incluye a las demás actividades (5) y otros en cambio, consideran que es el Alpinismo el que agrupa a todas las prácticas (6).

Desde los aportes de Moscoso (5), puede definirse a las actividades del Montañismo/Alpinismo a partir de cuatro modalidades de base: Alpinismo, Escalada, Esquí de travesía y Excursionismo. La primera involucra como submodalidades al alpinismo glacial, de dificultad, invernal, expedicionario, de altitud y ascensionismo; la segunda, a la Escalada deportiva, en hielo, artificial, big wall, escalada combinada, Boulder y sólo integral. La tercera incluye al Esquí de montaña/travesía y al Esquí alpino. En la última modalidad se considera al senderismo y trekking y raquetas de nieve.

De acuerdo al mismo autor se pueden establecer dimensiones fundamentales de los deportes de montaña, a partir de cuatro ejes: plano, terreno, estilo y estructura.

En relación con el plano, se distinguen: vertical, intermedio o combinado y horizontal; lo que permite ubicar al Alpinismo y a la Escalada en el vertical, al Esquí de montaña en el intermedio y al Excursionismo en el horizontal. La referencia sobre el terreno sitúa al tipo de lugar y orienta sobre la técnica a desarrollar. El Alpinismo incluye a la roca, la nieve, el hielo, el mixto y el artificial. El Esquí de montaña implica a la nieve y el Excursionismo al terreno rocoso, herboso y/o también helado.

El estilo determina al Alpinismo sobre el libre (se progresa sobre terreno en contacto directo y hay sistemas de aseguración ante una eventual caída) y el artificial (los sistemas de aseguración están unidos a la progresión, ya que el terreno supera la capacidad humana de desplazarse en contacto directo con la roca y/o el hielo). La estructura involucra a todas estas disciplinas, estableciendo algunas otras variables como la altitud, la intensidad y dificultad, las coordenadas temporales, la infraestructura, material, ayuda, conocimiento, las clasificaciones estándar y otras características particulares. El surgimiento del Alpinismo como deporte se vincula con la ascensión del Mont Blanc (4.807 mts.) en el año 1786. El término Alpinismo se utiliza en un principio sólo para definir la actividad montañera en los Alpes, pero luego se hace extensiva a la práctica desarrollada en todas las montañas. En la actualidad se nomina Alpinismo a la práctica en terreno nevado o de altura, caracterizándolo como montañismo de dificultad y se distingue claramente de la Escalada en terreno alpino o de aventura en alta montaña o montaña con hielo.

En síntesis, podría considerarse que el Alpinismo compromete a todas las actividades de la montaña, pero no a todas las personas que estén relacionadas con la naturaleza; pues, por ejemplo, quien realiza trekking no puede ser considerado alpinista. Socialmente, el montañismo sí incluye a todas las personas que desarrollan alguna de las actividades del alpinismo, denominándose también como montañeros. Desde el Plan de Estudios actual se plantea una orientación en prácticas de montañismo y escalada, aunque la revisión y propuesta de reformulación que se encuentra en proceso de elaboración propone definir a esta orientación como Prácticas de Andinismo.

\section{LAS PRÁCTICAS DE ANDINISMO EN LA FORMACIÓN DEL PROFESOR EN EDUCACIÓN FÍSICA}

El medio natural se convierte en ámbito de intervención pedagógica y en contenido educativo. Reclama un abordaje 
LÓPEZ E. \& FERNÁNDEZ M. Las actividades de andinismo en la formación del profesorado en educación física Rev. Motr. Hum.,

11(1): 6-10, 2010.

transdisciplinario que implica considerar saberes referidos a su comprensión e interpretación, visibilizando los vínculos que se establecen con él. Aporta posibilidades motrices que promueven el desarrollo de la corporeidad y motricidad, a partir de amplias y combinadas experiencias de movimientos que suponen el uso creativo del cuerpo.

En tanto escenario que favorece situaciones motrices específicas, culturalmente relevantes, se manifiesta como oportunidad para superar prácticas corporales estereotipadas, homogéneas, modélicas y disciplinadoras.

Uno de los desafíos planteados, vinculado con estas prácticas y en particular con las de Andinismo, es la selección de aquellas más relevantes para ser integradas a la formación del profesor en Educación Física. En este sentido, el diseño curricular que nos ocupa incluye el Trekking, la Escalada en roca, la Escalada deportiva en muro artificial y las Caminatas invernales con o sin raqueta.

Las prácticas motrices que fomentan estas actividades contribuyen a la formación integral, propician la autonomía de los sujetos y exigen el análisis y el reajuste constante de la conducta en relación con las situaciones percibidas y experimentadas. De igual manera, impulsan actitudes de cooperación y solidaridad desde la confianza en el otro y la admisión de la responsabilidad por el otro. Con el fin de evitar que los conocimientos permanezcan fragmentados, aislados y recortados, los contenidos de las materias se despliegan como un espiral a lo largo de las cursadas, otorgando continuidad y profundización en el tratamiento de las diversas temáticas. Los contenidos también se organizan respondiendo al principio de la progresión, aspecto que se refleja a partir del detalle que minuciosamente se efectúa en cada una de las asignaturas que conforman el trayecto orientado. En los tres espacios curriculares se proponen los siguientes ejes transversales de contenidos que relacionan e integran saberes: seguridad y prevención, equipos y materiales, cartografía y orientación, técnica y enseñanza en medios naturales, conciencia de conservación e interrelación con el medio.

La seguridad que requieren las prácticas de Andinismo resulta un aspecto básico y estructurante de las mismas y representa otro desafío en el proceso de formación. Tomando los aportes de Schubert (7), se instala el principio de redundancia que plantea el tener siempre un sistema alternativo de seguridad y también significa repensar la propia seguridad y la del otro. Por ejemplo, el contenido "tirolesa" se plantea desde el rescate en montaña, donde prevalece la máxima seguridad; una vez apropiado este concepto, se tendría la amplitud de armar una tirolesa de acuerdo a la práctica que se proponga. Es inagotable la cantidad de tirolesas que se podrían armar de acuerdo al propósito de las mismas y a las personas que la realizaran. De esta manera, en las propuestas pedagógicas es posible aplicar esta seguridad de acuerdo a las características y exigencias de la situación de enseñanza. Cada una de las asignaturas posee un riguroso protocolo de seguridad específico que manifiesta condiciones y requerimientos para las cursadas, entre los cuales se refiere la cantidad de alumnos por comisión, la cantidad de docentes por alumno, el itinerario previsto, los permisos y seguros pertinentes, las condiciones climáticas, el equipamiento básico y complementario, los pasos a seguir en caso de emergencias o accidentes.

Un último desafío que analizamos en esta oportunidad, se refiere a las incumbencias profesionales. Los alcances del título y las actividades para las que resulta competente el egresado de este profesorado, requieren mayores especificaciones ya que incluye actividades desarrolladas tradicionalmente por otros profesionales. En este aspecto, se presenta una problemática que reclama acciones articuladas con otras instituciones nacionales que titulan sobre actividades en la montaña, entre las cuales se encuentran la Asociación Argentina de Guías de Montaña (AAGM) de Bariloche; la Escuela Provincial de Guías de Alta Montaña y Trekking (EPGAMT) de Mendoza; la Escuela Tavien de Buenos Aires; la Universidad Nacional de Córdoba en convenio con la AAGM y el Centro Andino Buenos Aires. Por otro lado, diversos organismos de la región otorgan habilitaciones y reconocen las titulaciones anteriores: la Municipalidad de San Carlos de Bariloche, la provincia de Río Negro y Parques Nacionales vinculados a las zonas de montaña, instituciones que legislan estas actividades.

El campo laboral de los egresados se ha definido y redefinido desde los orígenes de la carrera. La Ordenanza N²641-1994 del Ministerio de Educación de la Nación, que refiere a los alcances del Título de Profesor en Educación Física que otorga la Universidad Nacional del Comahue, enuncia: -Planificar, conducir y evaluar procesos de enseñanza y de aprendizaje en el área de la Educación Física. - Asesorar en lo concerniente a los aspectos teóricosmetodológicos relativos a la enseñanza de la Educación Física.

-Conducir grupos en deportes y actividades de montaña de baja y mediana complejidad.

En este marco se definen deportes y actividades de montaña de baja y mediana complejidad al trekking con y sin nieve, hasta dificultad alta o de características similares; senderos y/o cumbres que no superen los 3.500 metros de altitud; prácticas de escalada en roca con cuerda de arriba, rapel escuela, cuerda fija, tirolesa de uso recreativo; prácticas en muro artificial con cuerda de arriba y mini - muro. En calidad de colaborador de Guía/s de Montaña, podrán realizar actividades de montaña hasta 4.500 metros de altura sobre nivel del mar en trekking, travesías y/o cumbres. Podrán trabajar en trekking y travesías con una relación docente/alumno de hasta 10 personas y no más de siete personas en prácticas de escalada en ambiente natural o artificial. 
LÓPEZ E. \& FERNÁNDEZ M. Las actividades de andinismo en la formación del profesorado en educación física Rev. Motr. Hum.,

11(1): 6-10, 2010.

\section{A MODO DE CIERRE}

La Educación Física ha sufrido cambios, otras demandas vinculadas con el entorno natural interpelan a la formación docente y generan nuevos desafíos y problemáticas en el proceso de formación.

La montaña se manifiesta como nuevo ámbito de intervención pedagógica. Las prácticas allí desplegadas orientan y generan situaciones motrices relevantes que forman la trama de la cultura de lo corporal y desencadenan saberes sustantivos para el desarrollo de competencias que promueven la emancipación de los sujetos.

A lo largo de los años transitados en la formación docente universitaria en Educación Física del CRUB, de la trayectoria recorrida y de los conocimientos construidos, las prácticas de andinismo se han ido legitimando en el universo simbólico de significación de la Educación Física. Aún es preciso profundizar en los aportes y las finalidades de estas prácticas, interrogadas desde los fundamentos de la educación.

\section{REFERENCIAS BIBLIOGRÁFICAS}

1. Arribas H. El pensamiento y la biografía del profesorado de Actividad Física en el medio natural. Un estudio multicaso en la formación universitaria orientado a la comprensión de modelos formativos. Universidad de Valladolid. Facultad de Educación y Trabajo Social, 2008.

2. Winter S. Escalada deportiva con niños y adolescentes. Editorial Desnivel. Munich. 2000.

3. Da Silva T. Descolonizar el currículum: estrategias para una pedagogía crítica. En Gentili P. (comp) Cultura, política y currículo. Losada. Buenos Aires. 1997.

4. Bracht V. Identidad y Crisis de la Educación Física: un enfoque epistemológico. En Bracht $\mathrm{V}$ y Crisorio R. (coordinadores) La Educación Física en Argentina y en Brasil. Identidad, desafios y perspectivas. Ediciones Al Margen. La Plata. 2003.

5. Moscoso D. La montaña y el hombre, en los albores del siglo XXI. Editorial Barrabés. Benasque. 2003.

6. Zorrilla J. Enciclopedia de la Montaña. Editorial Desnivel. Madrid. 2000.

7. Schubert. Seguridad y riesgo. Editorial Desnivel. Madrid. 1993. 
LÓPEZ E. \& FERNÁNDEZ M. Las actividades de andinismo en la formación del profesorado en educación física Rev. Motr. Hum.,

11(1): 6-10, 2010.

\section{ABSTRACT}

The work describes some challenges and problematic that appear in the formation of professorship in Physical Education of the Centro Regional Universitario Bariloche dependent on the Universidad Nacional del Comahue, Argentina; about demands linked with the natural environment. This career includes programs that promote the construction of corporeity and motricity from culturally relevant body practices in the natural environment, space that turns out to be a motivating potential, contributes to the integral formation from a boarding transdisciplinary and promotes the autonomy of subjects while it requires making of constant decisions in relation with his particular characteristics. The natural environment provides ample opportunities that exceed motor stereotypies, promotes the creative use of the body and enables new areas of educational intervention.

The activities of Mountaneering have been consolidated as contents programs, giving to this educational university particular formation characteristics, in narrow relation with the region.

Key words: Mountaneering, natural enviroment, physical education, educational formation.

\section{Dirigir correspondencia a:}

Eduardo López

Universidad Nacional del Comahue

Bariloche, Republica Argentina

Quintral S/No Bo. Jardín Botánico S/N (San Carlos De Bariloche)

E-mail: ehltato@yahoo.com

RECIBIDO 25-01-2010

ACEPTADO 27-03-2010 